\title{
Radiation-Stimulated Transformation of the Reflectance Spectra of Diazoquinone-Novolac Photoresist Films Implanted with Antimony Ions
}

\author{
A. A. Kharchenko ${ }^{a, b}, *$, D. I. Brinkevich ${ }^{a, * *}$, V. S. Prosolovich ${ }^{a}$, S. D. Brinkevich ${ }^{a}$, \\ V. B. Odzaev ${ }^{a}$, and Yu. N. Yankovski ${ }^{a}$ \\ ${ }^{a}$ Belarusian State University, Minsk, 220030 Belarus \\ ${ }^{b}$ Research Institute for Nuclear Problems, Belarusian State University, Minsk, 220030 Belarus \\ *e-mail:XaaTM@mail.ru \\ **e-mail:brinkevich@bsu.by
}

Received September 12, 2019; revised November 22, 2019; accepted November 25, 2019

\begin{abstract}
We measure the reflectance spectra of $1.8 \mu \mathrm{m}$-thick FP9120 photoresist films doped with antimony ions and deposited by centrifugation on the surface of $p$-type silicon wafers $(\rho=10 \Omega \mathrm{cm})$ with a (111) orientation. Implantation leads to a decrease in the refractive index of the photoresist due to the radiation crosslinking of Novolac resin molecules and a decrease in the molecular refraction and density of the photoresist. In the opacity region of the photoresist film, an increase in the reflection coefficient is observed with an increase in the implantation dose.
\end{abstract}

Keywords: lithography, radiation, substrate, photoresist, implantation, antimony, refractive index, reflection DOI: $10.1134 /$ S 1027451020030283

\section{INTRODUCTION}

The study of radiation-induced processes in polymers is of practical interest, since polymer compositions are actively used as a resist in electronic, photo, and X-ray lithography in the production of integrated circuits [1-8]. Currently, ion implantation is the primary doping method in microelectronics and nanoelectronics [9]. Increasing the degree of integration places high demands on the unit of operations that ensure masking of the ion beam. DiazoquinoneNovolac (DQN) resists play a significant role as masks in modern lithographic processes [1]. The interaction of DQN resists with far ultraviolet, X-ray, and visible radiation has been studied in sufficient detail [1]. However, the effect of ion implantation on the properties of these resists has not been studied enough, although radiation-induced processes occurring during ion implantation can have a significant impact on the quality of devices created.

The goal of the work is to study the radiation-stimulated transformation of the reflectance spectra of positive DQN resists upon the implantation of antimony ions.

\section{MATERIALS AND METHODS}

In the present work, we studied the radiationinduced processes upon the implantation of $\mathrm{Sb}_{+}$ions into films of industrial positive photoresist FP9120, which is a composite of photosensitive $O$-naphthoquinondiazide and phenol-formaldehyde resin. A photoresist film $1.8 \mu \mathrm{m}$ in thickness was applied by an industrial process on the surface of $p$-type silicon wafers ( $\rho$ $=10 \Omega \mathrm{cm}$ ) with a (111) orientation by centrifugation at a rotation speed of $1800 \mathrm{rpm}$. Before forming the photoresist film, the silicon wafers were subjected to a standard cycle of surface cleaning in organic and inorganic solvents. The centrifuge rotation time was $40 \mathrm{~s}$. After applying the photoresist to the working side of the silicon substrate, the film was dried for $50-55 \mathrm{~min}$ at a temperature of $88^{\circ} \mathrm{C}$. The thickness of the photoresist films was controlled mechanically using a Dectak profilometer. Deviations from the average value over the plate did not exceed $1 \%$ for all the samples studied. Implantation with $60-\mathrm{keV} \mathrm{Sb}^{+}$ions in the dose range of $1 \times 10^{15}-5 \times 10^{16} \mathrm{~cm}^{-2}$ at an ion current density of $j=4 \mu \mathrm{A} / \mathrm{cm}^{2}$ was carried out in a residual vacuum of $10^{-5} \mathrm{mmHg}$ using a Vezuvii-6 ion-beam accelerator.

The reflectance spectra of the photoresist-silicon structures were recorded in the range of $\lambda=210-1100 \mathrm{~nm}$ using a Proscan MC-122 single-beam spectrophotometer at room temperature with a resolution of no worse than $1 \mathrm{~nm}$. The reflectance spectra were measured using an attachment for measuring specular reflection, which makes it possible to change the 